\title{
Structural Shimming for High-Resolution Nuclear Magnetic Reso- nance Spectroscopy in Lab-On-A-Chip Devices ${ }^{\dagger}$
}

\author{
Herbert Ryan, ${ }^{a} \ddagger$ Alison Smith, ${ }^{a}$ and Marcel Utz ${ }^{a, *}$ \\ Received Xth XXXXXXXXXX 20XX, Accepted Xth XXXXXXXXX 20XX \\ First published on the web Xth $X X X X X X X X X X 200 X$ \\ DOI: 10.1039/b000000x
}

High-resolution proton NMR spectroscopy is well-established as a tool for metabolomic analysis of biological fluids at the macro scale. Its full potential has, however, not been realised yet in the context of microfluidic devices. While microfabricated NMR detectors offer substantial gains in sensitivity, limited spectral resolution resulting from mismatches in the magnetic susceptibility of the sample fluid and the chip material remains a major hurdle. In this contribution, we show that susceptibility broadening can be compensated even in the presence of substantial mismatch by including suitably shaped compensation structures into the chip design. An efficient algorithm for the calculation of field maps from arbitrary chip layouts based on Gaussian quadrature is used to optimise the shape of the compensation structure to ensure a flat field distribution inside the sample area. Previously, the complexity of microfluidic NMR systems has been restricted to simple capillaries to avoid susceptibility broadening. The structural shimming approach introduced here can be adapted to virtually any shape of sample chamber and surrounding fluidic network, thereby greatly expanding the design space and enabling true lab-on-a-chip systems suitable for high-resolution NMR detection.

\section{Introduction}

High-resolution nuclear magnetic resonance (NMR) spectroscopy is promising as a detection and readout technique in microfluidic lab-on-a-chip devices. It can quantify hundreds of metabolites in biological systems simultaneously and without destruction of the sample. NMR metabolomics ${ }^{1-3}$ could therefore be a valuable tool for chip-based cellular assays, with many potential applications in systems biology research as well as medical diagnostics.

The integration of microfluidic devices with magnetic resonance spectroscopy presents technical challenges related both to sensitivity and to spectral resolution ${ }^{4}$. Sensitivity is critical, since the sample amount is severely limited in microfluidic systems. Typically, this restricts practical applications to proton NMR due to their abundance and their large gyromagnetic ratio. However, the chemical shift range of proton signals is small (of the order of $10 \mathrm{ppm}$ ), requiring spectral resolution of at least $0.01 \mathrm{ppm}$ to allow effective analysis of complex mixtures. It has been shown that miniaturisation of the NMR inductive receiver to a size commensurate with the sample provides substantial gains in mass sensitivity. Micro-fabricated receiver structures can be directly integrated into lab-on-achip devices, and systems with limits of detection as low as

\footnotetext{
$0^{a}$ School of Chemistry, University of Southampton, Highfield Campus, Southampton SO17 1BJ, United Kingdom

$0 \ddagger$ Present address: Institut für Mikrotechnik (IMTEK), Albrecht-LudwigUniversität Freiburg, Germany.
}

$0.025 \mathrm{nmol} \cdot \mathrm{s}^{1 / 2}$ have been reported in the literature ${ }^{5}$. Different detector geometries and microfabrication strategies for microfluidic NMR have been described, including solenoids ${ }^{6-8}$, planar spirals $^{9-12}$, and microslots/micro striplines ${ }^{13-16}$. While these approaches yield similar sensitivity for a given probe volume, resolution in the vicinity of $0.01 \mathrm{ppm}$ has only been achieved with very simple, linear fluidic geometries (capillar-

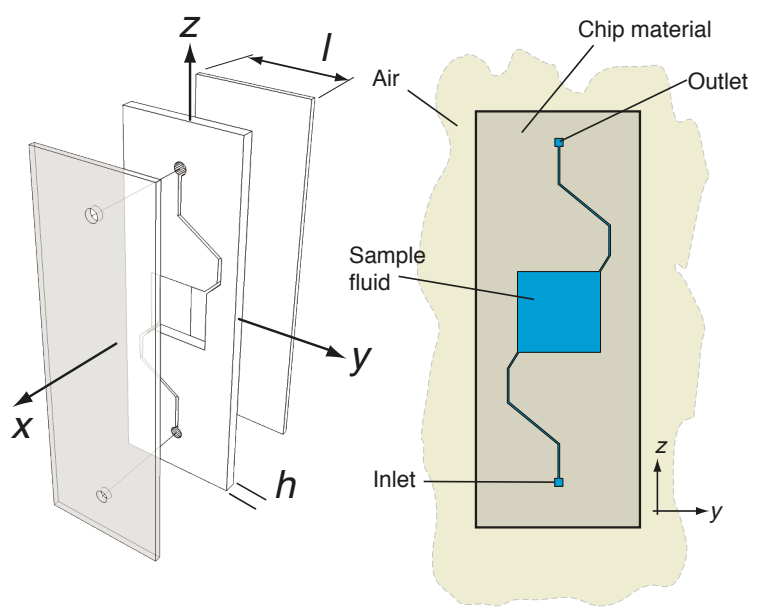

Fig. 1 Schematic drawing of a microfluidic chip consisting of a fludic layer sandwiched between a cover and a base layer. 

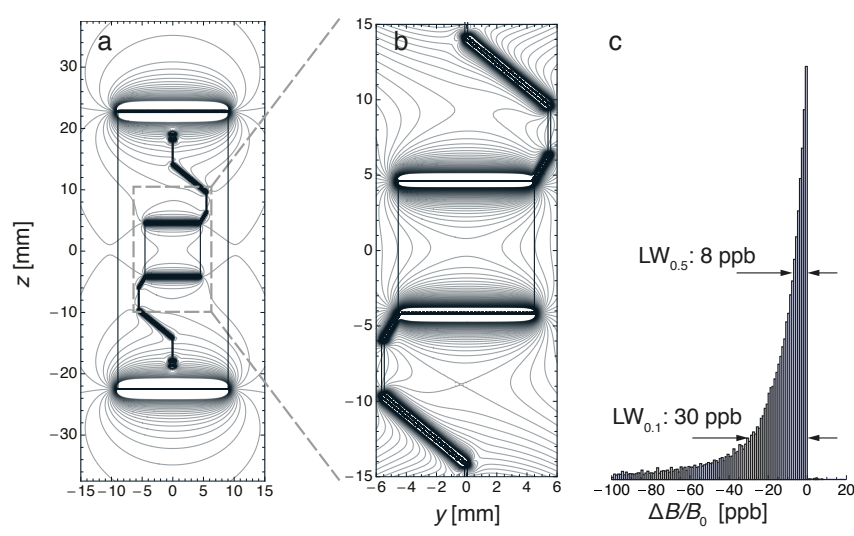

Fig. 2 Contour plot of the $z$ component of the computed demagnetising field for the chip shown in Fig. 1 with

$\chi_{\text {sample }}-\chi_{\text {chip }}=3 \mathrm{ppm}, 0.2 \mathrm{~mm}$ thickness of the fluidic layer, and $0.6 \mathrm{~mm}$ overall thickness of the chip. A: overview (contour spacing 20ppb); B: detailed view of the sample chamber (contour spacing: 2 $\mathrm{ppb}$ ); $\mathrm{C}$ : histogram of the demagnetising field values inside the sample chamber.

ies). By contrast, sample shapes with smaller aspect ratios such as circular or cylindrical chambers, while useful for microimaging, have not yielded satisfactory resolution for liquidstate proton NMR spectroscopy ${ }^{8,9}$.

Some examples for commonly used substrates in microfluidics are listed in Table 1. All are weakly diamagnetic, with volume susceptibilities in the range of $-11 \cdots-7 \mathrm{ppm}$ in SI units. The susceptibilities of common solvents vary in a similar range (cf. also Table 1). Therefore, depending on the choice of chip material and solvent, there is a mismatch between the microfluidic chip and the sample of one to several ppm. In general, this leads to variations of similar magnitude in the local magnetic field strength over the probe volume. For comparison, at common magnetic fields of several Tesla, the inherent line widths of many NMR signals in solution, particularly of protons in small molecules, are three orders of magnitude smaller. Spectral resolution of better than $0.001 \mathrm{ppm}$ is a typical specification for commercial highresolution NMR spectrometers operating with $5 \mathrm{~mm}$ diameter sample tubes. This is achieved by very careful design of the NMR probe system, together with adjustment of the currents through a multipolar shim set. Receiver coils are designed to excite only the central portion of the sample inside a cylindrical sample tube, thereby avoiding contamination of the signal from the air-sample interface. Special plugs with susceptibilities matched to common solvents are commercially available to handle situations where the available amount of sample is insufficient to fill a standard $5 \mathrm{~mm}$ tube. Due to the small dimensions of microfluidic systems (in the range of tens to hundreds of $\mu \mathrm{m}$ ), the spatial derivatives of the magnetic field are typically too large in magnitude to be compensated by the magnet shim coil set. Also, adjustment of the shims tends to be a time-consuming process, even though automated shim protocols have eliminated some of the tedium of the task.

In the present contribution, we show that it is possible to embed additional structures into microfluidic networks that compensate the field distortions, leading to good inherent field homogeneity even in the presence of substantial mismatches in susceptibility. The major advantage of this approach lies in its generality: it preserves the freedom of the designer, placing no a priori restrictions on the shape of the microfluidic network. Since the compensation structures can be fabricated by lithography at the same time as the fluidic network, they do not introduce additional fabrication overhead.

Fig. 1 shows a conceptual drawing of a microfluidic chip consisting of a fluidic layer with a square sample chamber, an inlet, and an outlet channel. The fluidic layer is sandwiched between a cover- and a base layer. Placed vertically in a homogeneous magnetic field, the horizontal surfaces of this structure cause an additional demagnetising field, as shown in Fig. 2. This leads to a continuous variation of the magnetic field inside the sample chamber. The resulting NMR line shape is given by the magnetic field histogram (Fig. 2C). For realistic dimensions and susceptibility values, the line width easily reaches $0.05 \mathrm{ppm}$, which is generally not acceptable for high-resolution proton NMR. As will be shown in the following, this distortion field can be compensated by adding suitably shaped air-filled structures to the chip design.

In the remainder of this paper, we first introduce an efficient computational method for calculating magnetic field distributions in planar structures. This approach is then used for the design of microfluidic chips with square sample chambers that yield high resolution even in the presence of $3 \mathrm{ppm}$

Table 1 Volume susceptibilities of materials commonly used for microfluidic devices and some common solvents

\begin{tabular}{lrr}
\hline \hline Material & $\chi_{V} \times 10^{6}$ & Ref \\
\hline PMMA & -9.02 & 17 \\
PDMS & -7.8 & 18 \\
Borosilicate glass & -11.0 & 18 \\
Teflon & -10.5 & 18 \\
\hline Solvent & $\chi_{V} \times 10^{6}$ & Ref \\
\hline Water & -9.05 & 18 \\
Deuterium oxide & -8.80 & 18 \\
Dimethyl sulfoxide & -8.30 & 18 \\
Ethanol & -7.29 & 18 \\
Methanol & -6.66 & 18 \\
\hline \hline
\end{tabular}




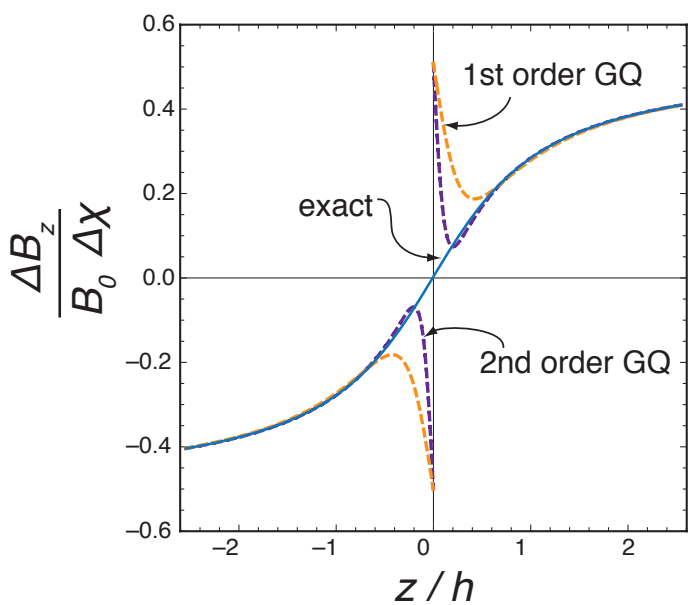

Fig. 3 Transition of the magnetic field across a rectangular boundary perpendicular to the field of depth $h$ and width $l=10 h$. Solid line: exact solution; dashed and dash-dotted lines: first and second order Gaussian quadrature, respectively.

mismatch in susceptibility. The resulting magnetic field distributions with and without the compensation structures are characterised using NMR micro-imaging and compared to the theoretical predictions. Finally, we use aqueous solutions of glucose to demonstrate the spectral resolution that can be achieved using compensation structures even in the presence of a strong susceptibility mismatch.

\section{Theory and Modelling}

Under stationary conditions and in the absence of macroscopic currents, Ampère's law dictates that $\nabla \times \boldsymbol{H}=0$, and the magnetic field $\boldsymbol{H}$ can therefore be expressed by a scalar magnetic potential $U$ as

$$
\boldsymbol{H}=-\nabla U
$$

In order to describe an object being inserted into an existing homogeneous magnetic field, we therefore split the potential as $U=U_{0}+U_{d}$, where $U_{0}=H_{0} z$ represents the homogenous original field, and $\boldsymbol{H}_{d}=-\nabla U_{d}$ is the field generated by the magnetic dipoles induced in the inserted object (often referred to as the demagnetising field). The magnetic field $H_{0}$, which we assume to be along the $z$-axis, typically originates from the persistent current in a superconducting solenoidal coil. The macroscopic magnetic induction is given by

$$
\boldsymbol{B}=\mu_{0}(\boldsymbol{H}+\boldsymbol{M}),
$$

where $\boldsymbol{M}$ is the induced magnetisation and $\mu_{0}=$ $4 \pi 10^{-7} \mathrm{Vs} /$ Am denotes the vacuum permeability. With
Gauss' law $\nabla \cdot \boldsymbol{B}=0$, one finds

$$
\nabla^{2} U_{d}=\nabla \cdot \boldsymbol{M}
$$

We assume that the object under study consists of a number of spatial domains characterised by a locally constant magnetic susceptibility $\chi_{k}$. The magnetisation is therefore piecewiseconstant,

$$
\boldsymbol{M}_{k}=\chi_{k} H_{0} \boldsymbol{e}_{z}
$$

and the right hand side of (3) vanishes everywhere except at the domain boundaries. The magnetic field satisfies the boundary conditions ${ }^{19}$

$$
\begin{aligned}
\left(\boldsymbol{H}_{d 2}-\boldsymbol{H}_{d 1}\right) \times \hat{\boldsymbol{n}} & =0, \\
\left(\boldsymbol{H}_{d 2}-\boldsymbol{H}_{d 1}\right) \cdot \hat{\boldsymbol{n}} & =H_{0}\left(\chi_{2}-\chi_{1}\right) \boldsymbol{e}_{z} \cdot \hat{\boldsymbol{n}},
\end{aligned}
$$

where $\hat{\boldsymbol{n}}$ denotes the surface normal (pointing from material 1 to material 2). Eqns. (3), (5), and (6) are formally solved by

$$
U_{d}(\boldsymbol{r})=\frac{H_{0}}{4 \pi} \int_{\partial_{12}} \frac{\hat{\boldsymbol{n}} \cdot \boldsymbol{e}_{z}\left(\chi_{2}-\chi_{1}\right)}{\sqrt{\left(\boldsymbol{r}-\boldsymbol{r}^{\prime}\right)^{2}}} \mathrm{~d} S
$$

where $\mathrm{d} S$ is an infinitesimal surface element, and $\boldsymbol{r}^{\prime}$ is the integration variable. If there are more than two different materials involved, each boundary gives and additive contribution of the same form. In our case, one of the domains is the sample, whereas the others represent the container surrounding it, as well as various elements of the probe assembly and radio frequency circuitry. The observed resonance frequency is proportional to the magnetic induction $\boldsymbol{B}_{\text {ext }}$ experienced by an ensemble of chemically equivalent nuclei within the sample domain. This induction is determined by the outside field $H_{0}$ plus the induced magnetic dipoles of all molecules in the sample domain except the one carrying the observed $\operatorname{spin}^{20}$. In liquids and isotropic solids, the external magnetic induction differs from the macroscopic $\boldsymbol{B}$ as

$$
\boldsymbol{B}_{\mathrm{ext}}-\boldsymbol{B}=-\frac{2 \mu_{0} \chi_{s}}{3} H_{0}
$$

which is often referred to as the induction generated by a hypothetical sphere of exclusion around the observed spin (Lorentz sphere). The magnetic induction relevant for the Larmor precession of nuclear spins in the sample is therefore

$$
\boldsymbol{B}_{\text {ext }}=\mu_{0} H_{0}\left(1+\frac{\chi_{s}}{3}\right) \boldsymbol{e}_{z}-\mu_{0} \nabla U_{d}
$$

Since $\chi$ is piecewise constant, only the last term leads to continuously varying fields, and therefore to line broadening, whereas the first term produces a bulk magnetic susceptibility shift (BMS) of the resonance line.

Microfluidic chips mostly consist of two-dimensional structures forming a network of fluidic channels etched into a substrate. We assume the plane of the chip to be aligned with the $z$ 
axis, and the side walls of the channels to be perpendicular to the base plane of the chip. In this case, the demagnetising field in the chip is caused purely by the channel side walls and by its outer edges. The top and bottom panels of the structure do not contribute since they are aligned with the field $\left(\hat{\boldsymbol{n}} \cdot \boldsymbol{e}_{z}=0\right)$. In many cases, the channel depth is smaller than the lateral dimensions of the chip. In this case, it is possible to integrate the contribution of a boundary segment to the demagnetising field over the layer thickness by Gaussian quadrature.

To illustrate this, consider a surface segment of length $l$ and depth $h$, oriented perpendicular to the magnetic field, separating two media with susceptibilities $\chi_{1}$ and $\chi_{2}$, respectively. $\chi_{2}-\chi_{1}=\Delta \chi$. The magnetic potential for such a segment is given by

$$
\begin{aligned}
& U_{d}(x, y, z)=\frac{\Delta \chi B_{0}}{4 \pi \mu_{0}} \times \\
& \times \int_{-h / 2}^{h / 2} d x_{1} \int_{-l / 2}^{l / 2} d y_{1} \frac{1}{\sqrt{\left(x-x_{1}\right)^{2}+\left(y-y_{1}\right)^{2}+z^{2}}} .
\end{aligned}
$$

In the present case, we restrict our discussion to the central $y z$ plane, such that $x=0$. Expression (10) can not be integrated symbolically. However, the integration over the length of the segment $\left(d y_{1}\right)$ can be carried out easily, to give

$$
\begin{aligned}
& U_{d}(x=0, y, z)=\frac{\Delta \chi B_{0}}{4 \pi \mu_{0}} \times \\
& \times \int_{-h / 2}^{h / 2} d x_{1} \ln \frac{2 y+l+\sqrt{(l-2 y)^{2}+4\left(x_{1}^{2}+z^{2}\right)}}{2 y-l+\sqrt{(l-2 y)^{2}+4\left(x_{1}^{2}+z^{2}\right)}} .
\end{aligned}
$$

The integrand in this expression has a singularity at the location of the segment for $x_{1}=0$, which disappears upon integration over the height $h$. It should be noted that the expressions under the square roots in the integrand vary slowly as a function of $x_{1}$ whenever $x_{1}^{2} \ll z^{2}$. Since $x_{1}$ is restricted to values between $\pm h / 2$, this is true for all values of $z$ that satisfy $z>h$. Therefore, the integral in (11) can be approximately evaluated by a low order Gaussian quadrature (GQ), which will give accurate results everywhere in the $y, z$-plane except where the distance from the segment is small compared to the segment height $h$.

This is illustrated in Fig. 3, which shows the normalised transition of the magnetic field across a horizontal boundary of length $l$ and height $h / l=0.1$. The exact result (which can be evaluated in closed form for the centre line $y=0$ ) is shown as a solid line, along with the results obtained from first and second order Gaussian quadrature (dashed and dash-dotted lines). While the actual transition of the magnetic field across the boundary is smooth and monotonous, the Gaussian quadrature approximations exhibit a remnant of the singularity in the

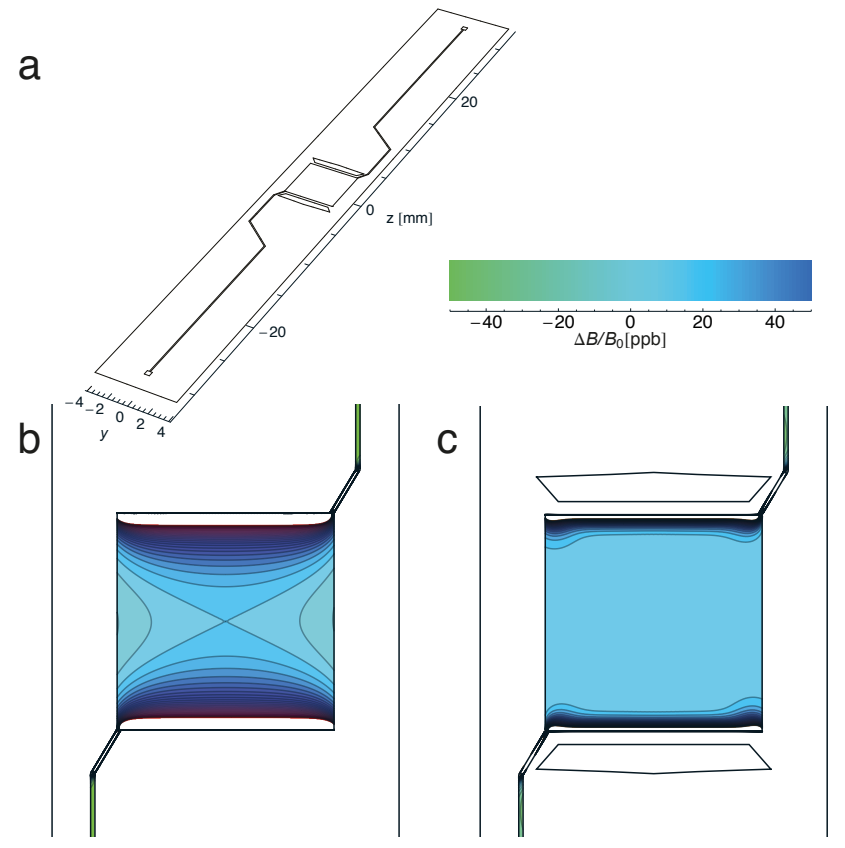

Fig. 4 a: Chip design used for NMR experiments. A central square sample chamber can be filled through the symmetrically arranged access/escape channels. Air-filled compensation structures are positioned on both sides of the sample chamber. b: field map resulting from $\chi_{\text {sample }}-\chi_{\text {chip }}=3$ ppm without compensation structures (contour spacing $0.01 \mathrm{ppm}$ ). c: same field map with air-filled compensation structures present.

integrand of eq. (11) near the boundary. However, first order GQ provides an excellent approximation to the magnetic field shape outside of $|z| / h>0.25$, whereas second order GQ is accurate to $|z| / h>0.1$. It should be noted that 1 st order GQ corresponds to a single evaluation of the integrand (at $\left.x_{1}=h / 4\right)$, whereas 2nd order GQ requires two (at $x_{1}=h(1 \pm \sqrt{3}) / 6$ ).

On the basis of these approximations, it is straightforward to derive an algorithm to predict the in-plane magnetic field distributions resulting from arbitrary lithographic structures. The geometry is defined in terms of a list of linear boundary segments, along with the change in magnetic susceptibility that each segment represents. Such lists are readily produced from CAD data. The field distribution is then computed by addition of the contributions from each segment by appropriate scaling, rotation, and translation of the demagnetising field caused by a single segment. Fig. 2 shows a contour plot of the demagnetising field in a microfluidic chip which has been obtained in this way, using a computer program written in Mathematica. 
Experiment
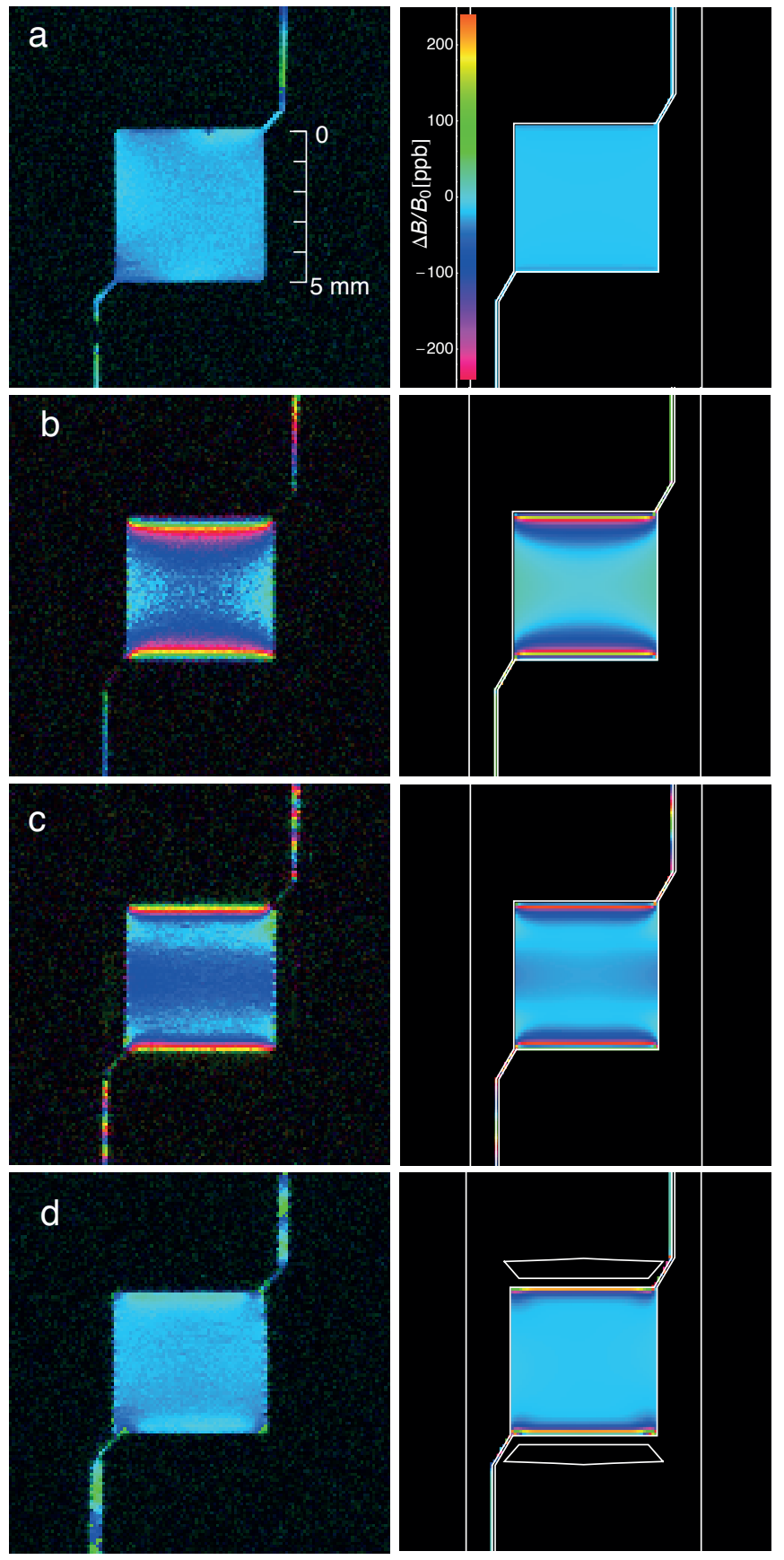

Simulation
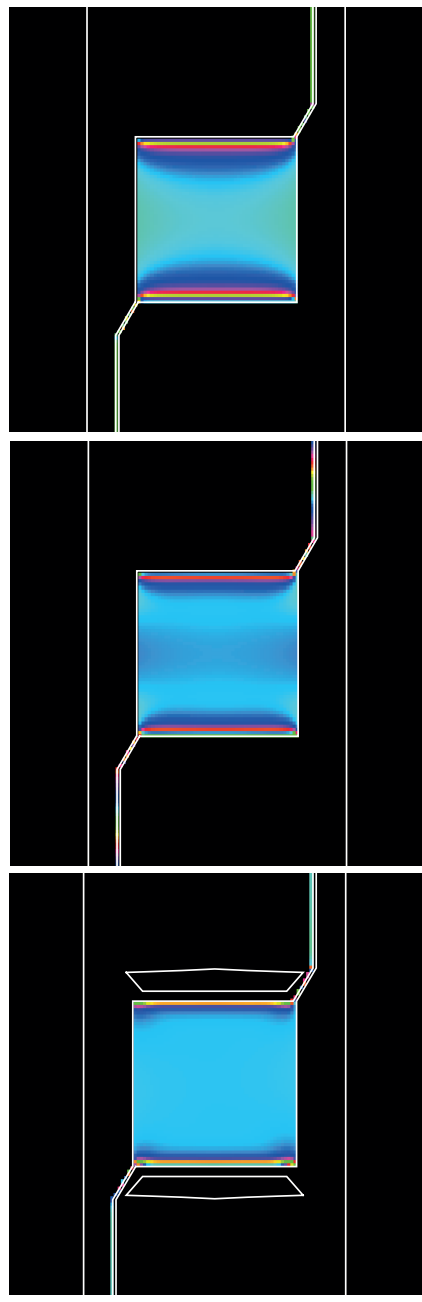

Fig. 5 Experimental (left column) and simulated (right column) $B_{0}$ field maps. a: DI $\mathrm{H}_{2} \mathrm{O}$ sample, chip without compensation structures; b: $52 \mathrm{mM} \mathrm{EuCl}_{3}$ in DI $\mathrm{H}_{2} \mathrm{O}$, no compensation structure; c: same as b, but first- and second-order shims optimised; d: same as b, but with air-filled compensation structures present in the chip design. The colour mapping is identical to the one in Fig. 4. The nominal dimensions of the chip are superimposed onto the simulated field maps (white lines).

\section{Experimental}

\subsection{Materials and Reagents}

All chemicals were purchased from Sigma-Aldrich unless otherwise stated. Microfluidic chips were fabricated from polymethyl methacrylate (PMMA) sheets (Weatherall Equipment \& Instruments Ltd, Buckinghamshire, UK). EuropiumIII-chloride was purchased from Aldrich was stored under moisture-free conditions, and used as received. Aqueous solutions were prepared using deionised (DI) water (Aldrich). Solutions of glucose for NMR were diluted from a $1 \mathrm{M}$ stock solution (from solid, $>99.5 \%$ ) prepared at least 24 hours before NMR measurements and stored at $4^{\circ} \mathrm{C}$.

\subsection{Microfluidic Chips}

Chip layouts were designed in Adobe Illustrator. The design was imported into Mathematica, and a custom-programmed function was used to predict the demagnetising fields as described in the theory section. The dimensions of the compensation structures were optimised manually, by adjusting their height and tip angle until the predicted line shape could not be improved any further. The resulting data set was then exported as a DXF file, and converted into a laser cutting tool path manually using CorelDraw (Corel), taking into account the cutting width of $120 \mu \mathrm{m}$, which had been determined by optical microscopy by cutting a test pattern. The fluidic layers were cut from PMMA sheet material of $200 \mu \mathrm{m}$ thickness using a Epi$\log$ Laser Mini $\mathrm{CO}_{2}$ cutter (Colorado, USA) with speed and power both set to $10 \%$. Top and bottom cover layers were cut in the same manner from $0.5 \mathrm{~mm}$ PMMA. The three layers were bonded together by wicking $5 \ldots 20 \mu 1$ of a mixture of $33 \%$ 1,2-dichloro ethane in $67 \% \mathrm{v} / \mathrm{v}$ ethanol in between the layers. ${ }^{21,22}$ The chips were then clamped between microscope slides using bulldog clips, and were left for $24 \mathrm{~h}$ at $65^{\circ} \mathrm{C}$. The fluidic channels were cautiously flushed with DI water prior to heat treatment, which prevented them from clogging. Bonded chips were flushed with $200 \mu 1100 \%$ ethanol (to minimise the formation of bubbles in the sample chamber) followed by $400 \mu \mathrm{l}$ DI water prior to filling with $200 \mu \mathrm{l}$ tap water or europium chloride solution for NMR. Following confirmation of a bubble-free chamber, the access channels of the chip were sealed using microamp clear adhesive film (Applied Biosystems).

\subsection{NMR Imaging and Spectroscopy}

All NMR spectra and images were obtained from a 7T Bruker AVANCEIII NMR spectrometer, equipped with a Bruker Micro20WB microimaging probe. The microfluidic chips were designed to provide a sliding fit inside standard $10 \mathrm{~mm}$ NMR sample tubes. They were roughly aligned and positioned in

This journal is @ The Royal Society of Chemistry [year]

Journal Name, 2010, [vol], 1-9 |5 


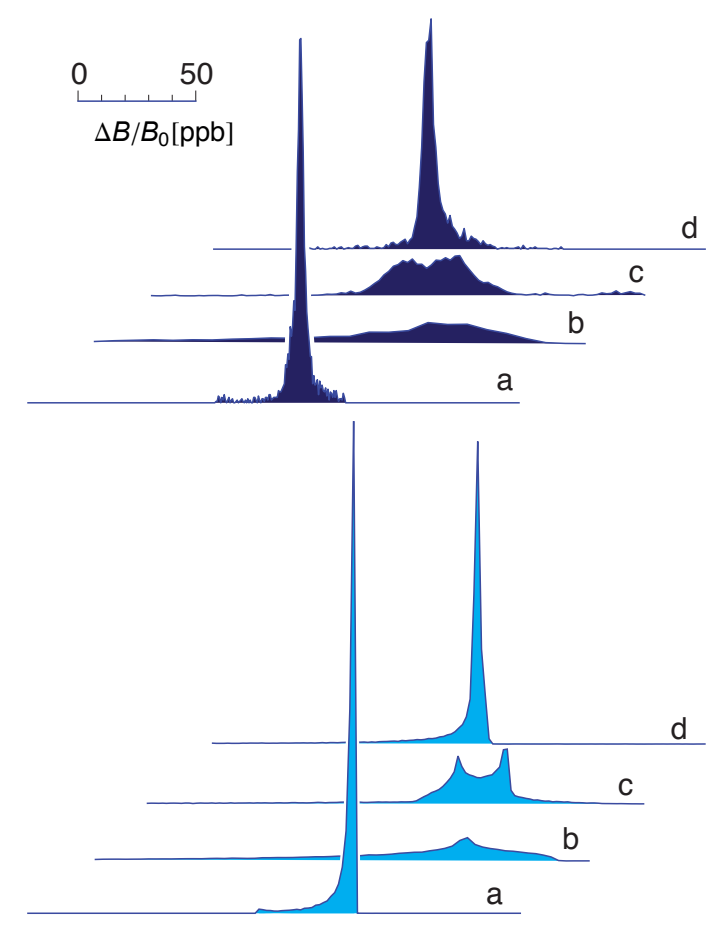

Fig. 6 Experimental (top) and simulated (bottom) $B_{0}$ histograms corresponding to the four cases a-d shown in Fig. 5. a: $\mathrm{H}_{2} \mathrm{O}$ sample, no compensation structures; b: $52 \mathrm{mMEuCl}_{3}$ in $\mathrm{DI} \mathrm{H}_{2} \mathrm{O}$, no compensation; c: same as b, after optimisation of shim settings; $d$ : same as b, but with compensation structures.

the probe manually. Fine positioning was then achieved by acquiring orthogonal scout images, and adjusting the probe height and orientation until the sample chamber was centred in the gradient coil system, and the chip normal was aligned with the imaging $x$-axis. $B_{0}$ field maps were obtained by acquiring two FLASH images at different effective echo times, and subtracting their phase values. The flip angle was $10^{\circ}$, and the repetition delay was set to $1 \mathrm{~s}$. Images were acquired at a resolution of $128 \times 128$ points with a field of view of $12.8 \mathrm{~mm}$ and a slice thickness of $1 \mathrm{~mm}$. 16 scans were averaged for each image. Glucose NMR spectra were acquired using the same probe, with a $90^{\circ}$ pulse length of $18 \mu$ s and a dwell time of $150 \mu \mathrm{s} .128$ scans were averaged with a relaxation delay of $5 \mathrm{~s}$. Spectra were manually phased and processed with $1 \mathrm{~Hz}$ of Lorentzian line broadening.

\section{Results and Discussion}

In order to demonstrate the principle of structural shimming experimentally, we have used microfluidic chips shown in Fig. 4a. The design consists of a square sample chamber of
$5 \mathrm{~mm} \times 5 \mathrm{~mm}$ size, connected to inlet and outlet channels at opposite corners. Variants of the chips were made both with and without the additional trapezoid-shaped structures shown in Fig. $4 \mathrm{a}$ at the top and bottom of the sample chamber. The fluidic structure was cut from PMMA sheets of $0.2 \mathrm{~mm}$ thickness. The resulting fluidic layers were solvent bonded ${ }^{21,22}$ to bottom and top covers of $0.5 \mathrm{~mm}$ thickness, as described in the experimental section.

Fig. $4 \mathrm{~b}$ and $\mathrm{c}$ show calculated contour maps of the demagnetising field inside the sample chamber assuming +3 ppm susceptibility mismatch of the sample with respect to PMMA. The top and bottom edges of the sample chamber act as sources for the demagnetising field. In the absence of compensating air-filled structures (Fig. 4b), this leads to a pronounced continuous variation of the magnetic field over the sample chamber; the resulting NMR line shape is broadened over more than $100 \mathrm{ppb}$. By contrast, in the chip with the air-filled compensation structures the demagnetising field is constant over almost the entire sample chamber within less than $10 \mathrm{ppb}$ (Fig. 4c). The shape of the compensation structures (average height and centre height) has been optimised by hand using a Mathematica model for the demagnetising field.

These predictions are confirmed by the experimental MRI field maps shown in Fig. 5. The first three rows (a-c) show results from uncompensated chips, whereas the last one is from a chip including compensation structures. The magnetic susceptibility of pure water at $20^{\circ} \mathrm{C}$ is $-9.05 \mathrm{ppm}^{18}$, very close to that of PMMA $\left(\chi=-9.01 \mathrm{ppm}^{17}\right)$. As a result, a flat field map with variations of less than $0.01 \mathrm{ppm}$ can easily be obtained in the uncompensated chip by adjusting the first- and second-order shim currents (Fig. 5a). Optimum values for the shim currents have been obtained by fitting a field map with a linear combination of the shim model functions. The shim model had been calibrated by measuring the changes in $2 \mathrm{D}$ field maps from a $10 \mathrm{~mm}$ sample tube filled with water upon incrementing the currents through individual shim channels. The residual variations in Fig. 5a result from slight warping of the chip bottom and top layers, which is caused by buckling due to residual stresses induced by the solvent-bonding process.

The bulk susceptibility of aqueous solutions can be varied across a wide range by adding lanthanide salts ${ }^{23}$. In the present work, we have used $\mathrm{EuCl}_{3}$ for this purpose. Complexes of $\mathrm{Eu}^{3+}$ are widely used as shift reagents in solution NMR spectroscopy ${ }^{24}$. As opposed to other lanthanides, $\mathrm{Eu}^{3+}$ has only a minimal effect on nuclear relaxation rates. Fig. $5 b$ (left) shows the field map obtained from the same chip after adding $52 \mathrm{mM} \mathrm{EuCl}_{3}$ to the sample. The demagnetising field profile is in very good agreement with the theoretical prediction (right hand column in Fig. 5). Shim currents have not been changed between Fig. 5a and b. Optimisation of the first- and second-order shims leads to the field profile shown 
in Fig. 5c. While the field variation in the transverse direction is eliminated by the shims, a substantial dependence in the $z$ direction remains. In principle, this could be addressed by using shim coils of higher order. However, their range is too small in practice to make much of a difference over the small sample size. By contrast, the field distortions are mostly eliminated by the inclusion of structural shimming structures in the chip (Fig. 5d).

Histograms of the demagnetising field inside the sample chamber are easily derived from the field map data. They reflect the effect of the demagnetising field on the NMR line shape. Fig. 6 compares the experimental histograms obtained from the data shown in Fig. 5 with the theoretical predictions, showing excellent agreement. While the small mismatch between water and PMMA leads to a relatively sharp line (Fig. 6a), a 3 ppm mismatch produces a very severely broadened line (b). Adjusting the shim currents improves this somewhat (c), but the line shape remains irregular, and still spans more than $20 \mathrm{ppb}$. Only in the presence of the air-filled shim structures is a line width of less than $10 \mathrm{ppb}$ recovered (Fig. 6d). It should be noted that the experimental histograms exhibit a foot on the high-field side (particularly a and d), which is not present in the simulations. This is an artefact due to slight out-of-plane warping of the micro fabricated chips.

Fig. 7 shows the effect of these line widths on the resolution in NMR spectra of aqueous solutions of glucose. The spectra have been acquired using the Bruker MicroWB20 probe coil with a proton/carbon insert like the field maps shown above. Solvent suppression was achieved by $\mathrm{CW}$ irradiation of the water resonance during the repetition delay, which was set to 12 seconds. A solution of glucose in DI water, without addition of $\mathrm{EuCl}_{3}$, yields a line width of about 0.01 ppm (corresponding to $3 \mathrm{~Hz}$ at $7 \mathrm{~T}$ ) in a PMMA chip without compensation structures. As can be seen in Fig. 7a, this is sufficient to resolve homonuclear $J$-couplings in the glucose spectrum. Note that all glucose solutions have been left for at least $24 \mathrm{~h}$ after preparation in order to reach equilibrium between the $\alpha$ and $\beta$ pyranose isomers. The doublets corresponding to the anomeric protons of both isomers are clearly resolved.

Addition of $52 \mathrm{mM} \mathrm{EuCl}_{3}$ to the solution (corresponding to +3 ppm susceptibility mismatch with the chip material) leads to very severe line broadening (spectrum not shown). The resolution can be partly restored by careful adjustment of the shim currents (Fig. 7b). However, most of the $J$-couplings are no longer resolved. Shimming in this case is subject to a tradeoff between the half-width of the line and the severity of its foott. The spectrum shown in Fig. $7 \mathrm{~b}$ corresponds to the field map in Fig. 5c, where optimal shim currents have been determined by fitting of the field profile prior to shimming.

A spectrum of similar quality to the one obtained in the susceptibilitiy-matched case is only retrieved when compensation structures are included in the chip design, as shown in
Fig. 7c. The line width in this case is about $6 \mathrm{~Hz}$, slightly larger than in the matched case. In spite of the similar resolution, there are some differences between the spectra Fig. 7a and c. It should be noted that since $\mathrm{Eu}^{3+}$ is a strong Lewis acid, the mismatched glucose solution is very acidic $(p \mathrm{H}<2)$. Since the $\mathrm{pH}$ is known to affect the anomeric equilibrium, ${ }^{25}$ this may explain the disappearance of the anomeric proton signal at $5.3 \mathrm{ppm} . \mathrm{EuCl}_{3}$ is not soluble at physiological $\mathrm{pH}$ and therefore the $\mathrm{pH}$ was not adjusted in these experiments. Another difference can be observed in the broad signals somewhat upfield from the solvent resonance. These features were found to depend strongly on the choice of rf power for solvent suppression. We therefore suspect that they stem from imperfect suppression of the water signal in the access channels of the chip, where the local field deviates strongly from its value in the centre of the sample chamber.

The experimental results presented above are in excellent agreement with the predictions from two-dimensional computation of the demagnetising fields based on second order Gaussian quadrature. As expected, significant deviations from the theoretical predictions in the field maps are confined to a region of less than $0.2 \mathrm{~mm}$ from the boundaries, where the

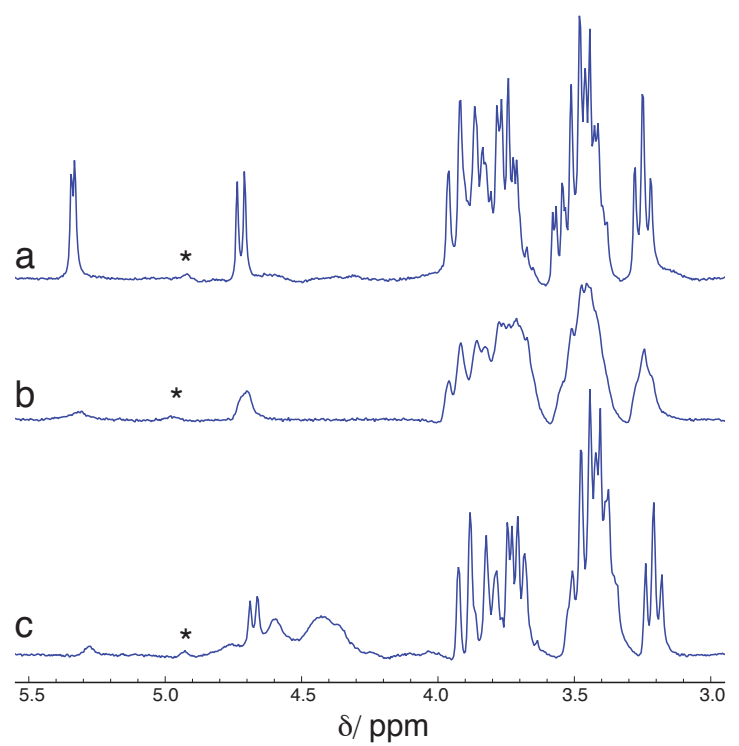

Fig. 7 Water-suppressed ${ }^{1} \mathrm{H}$ NMR spectra of glucose solutions in microfluidic chips with 5 x $5 \mathrm{~mm}$ sample chambers of $0.2 \mathrm{~mm}$ depth. a: $500 \mathrm{mM}$ glucose in DI $\mathrm{H}_{2} \mathrm{O}$; b: $52 \mathrm{mM} \mathrm{EuCl}_{3}$ and 500 $\mathrm{mM}$ glucose in DI $\mathrm{H}_{2} \mathrm{O}$, after optimisation of first- and second-order shim currents (corresponding to case c in Fig. 5 and Fig. 6); c: same as $b$, but in a chip equipped with air-filled compensation structures. The position of the suppressed water peak is indicated by asterisks. The water resonance was used as an internal chemical shift reference at $4.9 \mathrm{ppm}$. 

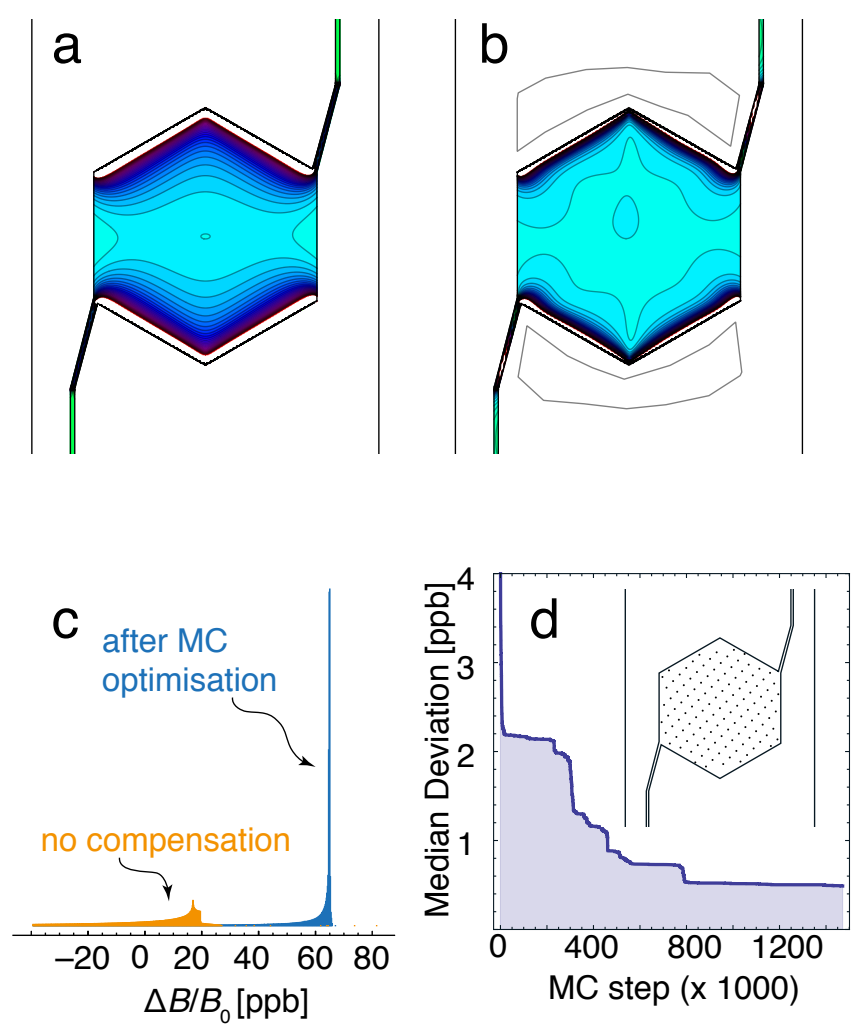

Fig. 8 Monte Carlo optimisation of a compensation structure. a: computed field map resulting from a hexagonal sample chamber of $5 \mathrm{~mm}$ diameter and $0.2 \mathrm{~mm}$ thickness, with $3 \mathrm{ppm}$ susceptibility mismatch between sample fluid and chip material. b: computed field map with optimised compensation structure; c: field histograms before and after optimisation; d: convergence of the median deviation of the field distribution. The inset shows the field sampling points that were used for the optimisation.

integration over the thickness dimension is less accurate.

The concept of structural shimming as presented here relies on the inclusion of air-filled structures. Since air is more paramagnetic $\left(\chi_{\text {air }}=0.2 \mathrm{ppm}^{23}\right)$ than typical chip materials, such compensation structures always produce contributions to the demagnetising field that are convex, i.e., the demagnetising field decreases with distance from the compensation structure. While, as it has been shown, they can compensate accurately the field distortions caused by a sample that is more paramagnetic than the chip, the opposite case cannot be dealt with in the same way. This would require filling the compensation structure with a medium that is substantially more diamagnetic than the chip material. Unfortunately, there are very few examples of liquids that meet this criterion. A possible solution would consist of adding an additional layer to the chip such that compensation structures could be placed directly on top of the fluidic structure of interest; however, this considerably increases the chip's complexity.

From the standpoint of structural shimming, it is therefore advisable to choose a chip material whose susceptibility either matches that of the sample fluid, or which is more diamagnetic. In this case, a flat field distribution can be achieved by including appropriately shaped compensation structures into the design. While the appropriate shape of the compensation structure has been adjusted by hand in the results presented above, it is straightforward to implement an automated optimisation procedure. As an example, Fig. 8 shows a simulation of a hexagonal sample chamber. The field distribution without compensation structures is shown in Fig. 8a. Monte Carlo optimisation of the compensation structures leads to a substantially improved field distribution (Fig. 8b). The improvement in line shape is also evident in the histograms (Fig. 8c). At each cycle of the Monte Carlo process, the vertices of the compensation structures are moved in the $y, z$-plane by a random amount, and the median deviation of the field distribution is recomputed using the sampling points shown in Fig. 8d. Only Monte Carlo steps that lead to an improvement (i.e., smaller median deviation of the field) are accepted. As shown in Fig. 8d, the procedure converged within $1.5 \times 10^{6}$ MC steps, requiring $1 \mathrm{~h}$ of CPU time on a desktop computer running $2.7 \mathrm{GHz}$ Intel Core $\mathrm{I} 5$ processor.

The present work has focused entirely on the inhomogeneities caused by the chip. In practice, microfluidic NMR systems require a metallic resonator surrounding the sample in order to couple in radio frequency signals. These probe structures can themselves cause susceptibility broadening. However, the demagnetising fields generated can be minimised by choosing planar geometries aligned with the magnetic field such as micro-stripline probes ${ }^{13-16}$. In general, the thickness of metallic detection structures can be reduced down to a few times the skin depth, which is of the order of a few $\mu \mathrm{m}$ for copper at typical NMR frequencies. Since the demagnetising fields scale linearly with the thickness of planar structures, this means that they can be reduced to very small levels. By contrast, this is not possible for the fluidic part of the system without concomitantly reducing the amount of sample.

\section{Conclusions}

In summary, we have shown that the distortions due to magnetic susceptibility differences in microfluidic systems can be modelled effectively in a two-dimensional manner. Spectral resolution of better than $0.01 \mathrm{ppm}$ can be achieved even in the presence of substantial susceptibility differences between the substrate material and the sample by including appropriately designed compensation structures into the chip design. For simple geometries, the shape of the compensation structure can be optimised manually. Monte Carlo optimisation can be 
implemented in order to deal with more complicated sample chamber geometries. Air-filled compensation structures can also be included in the design to mask long-range susceptibility effects caused by structures in the probe, external to the chip.

\section{Acknowledgement}

This work has been partially supported by the U.S. National Science Foundation under grant number CHE-0809795, by the U.S. National Institute of Health under award number 5R01EB011591, and by a Marie Curie career integration award (project uF-NMR) to MU from the 7th EU Framework Programme. We are indebted to Prof. Jan Korvink for helpful discussions. MU gratefully acknowledges a visiting fellowship from the Freiburg Institute of Advanced Study.

\section{References}

1 J. K. Nicholson and J. C. Lindon, Nature, 2008, 455, 1054-1056.

2 M. Čuperlović-Culf, NMR Metabolomics in Cancer Research, Woodhead Pub Limited, Cambridge, 2013.

3 T. W.-M. Fan, A. N. Lane and R. M. Higashi, The Handbook of Metabolomics, Humana PressInc, 2012.

4 V. Badilita, R. C. Meier, N. Spengler, U. Wallrabe, M. Utz and J. Korvink, Soft Matter, 2012, 8, 10583-10597.

5 D. A. Seeber, R. L. Cooper, L. Ciobanu and C. H. Pennington, Review of Scientific Instruments, 2001, 72, 2171.

6 D. L. Olson, T. L. Peck, A. G. Webb, R. L. Magin and J. V. Sweedler, Science, 1995, 270, 1967-1970.

7 D. L. Olson, M. E. Lacey and J. V. Sweedler, Analytical Chemistry, 1998, 70, 645-650.

8 V. Badilita, K. Kratt, N. Baxan, M. Mohmmadzadeh, T. Burger, H. Weber, D. v. Elverfeldt, J. Hennig, J. G. Korvink and U. Wallrabe, Lab Chip, 2010, 10, 1387-1390.

9 C. Massin, G. Boero, F. Vincent, J. Abenhaim, P.-A. Besse and R. Popovic, Sensors and Actuators A: Physical, 2002, 9798, $280-288$.

10 C. Massin, F. Vincent, A. Homsy, K. Ehrmann, G. Boero, P. A. Besse, A. Daridon, E. Verpoorte, N. F. de Rooij and R. S. Popovic, Journal of Magnetic Resonance, 2003, 164, 242-255.

11 K. Ehrmann, K. Pataky, M. Stettler, F. M. Wurm, J. Brugger, P.-A. Besse and R. Popovic, Lab Chip, 2007, 7, 381383.

12 H. Ryan, S.-H. Song, A. Zaß, J. Korvink and M. Utz, Anal. Chem, 2012, 84, 3696-3702.

13 Y. Maguire, I. L. Chuang, S. Zhang and N. Gershenfeld,
Proceedings of the National Academy of Sciences, 2007, 104, 9198-9203.

14 H. G. Krojanski, J. Lambert, Y. Gerikalan, D. Suter and R. Hergenröder, Analytical Chemistry, 2008, 80, 86688672.

15 J. Bart, A. J. Kolkman, A. J. Oosthoek-de Vries, K. Koch, P. J. Nieuwland, H. J. W. G. Janssen, J. P. J. M. van Bentum, K. A. M. Ampt, F. P. J. T. Rutjes, S. S. Wijmenga, H. J. G. E. Gardeniers and A. P. M. Kentgens, Journal of the American Chemical Society, 2009, 131, 5014-5015.

16 J. Bart, J. W. G. Janssen, P. J. M. van Bentum, A. P. M. Kentgens and J. G. E. Gardeniers, Journal of Magnetic Resonance, 2009, 201, 175-185.

17 Å. Carlsson, G. Starck, M. Ljungberg, S. Ekholm and E. Forssell-Aronsson, Magn Reson Imaging, 2006, 24, 1179-1185.

18 F. Doty, G. Entzminger and Y. A. Yang, Concepts in Magnetic Resonance, 1998, 10, 133-156.

19 J. D. Jackson, Classical Electrodynamics, John Wiley \& Sons, 3rd edn., 2007.

20 M. H. Levitt, Concepts Magn. Reson., 1996, 8, 77-103.

21 C.-H. Lin, C.-H. Chao and C.-W. Lan, Sensors and Actuators B: chemical, 2007, 121, 698-705.

22 C. R. Reedy, C. W. Price, J. Sniegowski, J. P. Ferrance, M. Begley and J. P. Landers, Lab on a Chip, 2011, 11, 1603-1611.

23 C. J. G. Bakker and R. de Roos, Magn. Reson. Med., 2006, 56, 1107-1113.

24 J. K. M. Sanders and D. H. Williams, Nature, 1972, 240, 385-390.

25 V. S. R. Rao and J. F. Foster, J. Phys. Chem., 1965, 69, 636-640.

This journal is @ The Royal Society of Chemistry [year]

Journal Name, 2010, [vol], 1-9 |9 